\title{
邻三氟甲磺酸酯基取代二芳基碘盐的合成及其抑菌活性研究
}

\author{
崔丽媛 ${ }^{a}$ 陈煌冠 ${ }^{a}$ 马静怡 ${ }^{a}$ 韩建伟 $*, a, b$ 王利民*,a \\ ( ${ }^{a}$ 华东理工大学化学与分子工程学院 上海 200237) \\ $\left({ }^{b}\right.$ 中国科学院上海有机化学研究所 沪港化学合成联合实验室 上海 200032)
}

\begin{abstract}
摘要 二芳基碘盐具有潜在的生物活性. 合成了 13 种邻位三氟甲磺酸酯基取代的线性二芳基碘盐, 利用核磁共振谱和 质谱表征了它们的结构. 通过微量肉汤稀释法测试该系列化合物的最低抑菌浓度(MIC), 结果表明含氟基团取代的二 芳基碘盐对于大肠杆菌(E.coli)、金黄色葡萄球菌(S. aureus)和枯草芽孢杆菌(B. subtilis)具有良好的抑菌性. 其中, 化合 物(3-氟苯基)(2-(( 三氟甲基)磺酰基)氧基)苯基)三氟甲磺酸碘盐(1)对大肠杆菌、金黄色葡萄球菌和枯草芽孢杆菌的 MIC 值分别为 16,4,4 $\mu \mathrm{g} \cdot \mathrm{mL}^{-1}$, 明显低于市售抗菌剂卡松(异噻唑啉酮). 此外, 扫描电子显微镜(SEM)结果显示, 该二芳基 碘盐能够明显诱导细菌死亡; 细胞试验结果显示在体外条件下, 化合物 1 与市售卡松对 Hela 细胞的生长影响相当, 是 一种低毒性的抗菌剂.
\end{abstract}

关键词 二芳基碘盐；抗菌剂；卡松；含氟化合物；三氟甲磺酸酯

\section{Synthesis of Vicinal Trifluoromethanesulfonate Substituted Diaryliodonium Salts and Their Anti-bacterial Properties}

\author{
Cui, Liyuan ${ }^{a} \quad$ Chen, Huangguan ${ }^{a} \quad$ Ma, Jingyi $^{a} \quad$ Han, Jianwei ${ }^{*, a, b}$ Wang, Limin ${ }^{*, a}$ \\ $\left({ }^{a}\right.$ School of Chemistry \& Molecular Engineering, East China University of Science and Technology, Shanghai 200237) \\ $\left({ }^{b}\right.$ Shanghai-Hong Kong Joint Laboratory in Chemical Synthesis, Shanghai Institute of Organic \\ Chemistry, Chinese Academy of Sciences, Shanghai 200032)
}

\begin{abstract}
Diaryliodonium salts with fluorines have potential bioactivity. Vicinal trifluoromethanesulfonate substituted diaryliodonium salts were synthesized for the purpose of studying their anti-bacterial properties aganist Escherichia coli (E. coli), Staphylococcus aureus (S. aureus) and Bacillus subtilis (B. subtilis). Firstly, their chemical structures were characterized by ${ }^{1} \mathrm{H}$ NMR, ${ }^{13} \mathrm{C}$ NMR, ${ }^{19} \mathrm{~F}$ NMR and mass spectra. The minimum inhibitory concentration (MIC) of these compounds was tested by the micro-broth dilution method. The most active 2-((3-fluorophenyl)(((trifluoromethyl)sulfonyl)oxy)-13-iodanyl)- phenyl trifluoromethanesulfonate (1) containing fluorine group gave the promising results for Escherichia coli (E. coli), Staphylococcus aureus (S. aureus) and Bacillus subtilis (B. subtilis) (MIC values of 16,4 and $4 \mu \mathrm{g} \bullet \mathrm{mL}^{-1}$ ), which is significantly lower than the commercial antibacterial agent of isothiazolinones. Scanning electron microscope (SEM) analysis sugggested that the compound led to the death of bacteria. Cytotoxicity tests showed that the compound $\mathbf{1}$ as well as isothiazolinones had a slight effect on the growth of Hela cells in vitro conditions.
\end{abstract}

Keywords diaryliodonium salts; anti-bacterial; Kathon; fluorine; trifluoromethanesulfonate

二芳基碘盐是一类典型的三价碘化合物, 因其碘正 离子高度缺电子性和碘苯的较易离去性, 在各种有机合 成反应中是良好的芳基化试剂 ${ }^{[1]}$. 最近, 本课题组发现 了一种温和的氧化方法, 可以制备一系列新颖的邻位三 氟甲磺酸酯取代的二芳基碘鎓盐; 基于此鎓盐，发展了
一种通过分子内芳基迁移而合成邻碘二芳基醚的新方 法 ${ }^{[2]}$.

近年来, 二芳基碘盐的催化性能和生物活性的研究 逐渐受到人们的关注 ${ }^{[3 \sim 5]}$. 例如, Shibata 小组报道了多 位点氟取代的二芳基碘盐对淋巴瘤细胞(U937)有明显

\footnotetext{
* Corresponding authors. E-mail: jianweihan@ecust.edu.cn; wanglimin@ecust.edu.cn

Received August 31, 2018; revised November 20, 2018; published online December 17, 2018

Dedicated to Professor Qingyun Chen on the occasion of his 90th birthday.

Project supported by the National Natural Science Foundation of China (Nos. 21472213, 21772039), the National Key Research and Development Program (No. 2016YFA0200302), and by the Croucher Foundation (Hong Kong) in the Form of CAS-Croucher Foundation Joint Laboratory Grant.

国家自然科学基金 (Nos. 21472213, 21772039)、国家重点研发计划(No. 2016YFA0200302)和香港雸搓基金会资助项目.
} 
的细胞毒性; 其中间位 $\mathrm{SF}_{5}$ 和氟多位点取代的二芳基碘 盐在 $5 \mu \mathrm{m}$ 的浓度下表现出 $78 \% \sim 94 \%$ 的细胞毒性. 邻 位取代二芳基碘盐表现出更高的细胞毒性, 邻位 $\mathrm{SF}_{5}$ 取 代的二芳基碘盐在 $5 \mu \mathrm{m}$ 的浓度下表现高达出 $98.9 \%$ 的 细胞毒性, 甚至在 $1 \mu \mathrm{m}$ 的低浓度下的细胞毒性也达到 $66.9 \%$; 邻位 $\mathrm{CF}_{3} \mathrm{SO}_{2}$ 取代的二芳基碘盐在 $5 \mu \mathrm{m}$ 的浓度 下表现出高达 $98.6 \%$ 的细胞毒性 ${ }^{[6]}$. 值得注意的是, 邻 位取代的二芳碘盐化合物在 $5 \mu \mathrm{m}$ 的浓度下对正常人类 细胞(AGLCL)仅约为 50\%的中等毒性; 这种对肿瘤细胞 和正常细胞的毒性选择性差异为抗肿瘤药物的研发提 供了新思路. 因此, 邻位取代的二芳基碘盐的生物活性 引起了格外的注意. 此外, Goldstein 等 ${ }^{[7]}$ 和 Doroshow 等 ${ }^{[8]}$ 分别报道了二芳基碘盐具有体外抗菌活性. Goldstein 的研究发现, 具有不同长度疏水链和含有单或双的 碘正离子的碘盐化合物对口腔细菌均具有广谱杀菌性, 与防腐消毒药物双氯苯双胍已烷的杀菌性相当, 适合应 用于漱口水等口腔卫生用品.

工业抗菌剂分为氧化型和非氧化型两类, 卡松是常 见的异噻唑啉酮类非氧化型工业抗菌剂, 具有低毒、高 效等优点, 但是存在着大量使用后, 导致微生物产生耐 药性等问题. 因此, 设计更有效的新型抗菌剂并有效地 克服微生物耐药性成为科学家研究的重点. 高价卤素具 有强氧化性, 含高价卤素原子的化合物因氧化性可以有 效地杀死葡萄球菌、大肠杆菌和绿脓假单胞菌等常见致 病菌, 且极少产生耐药性 ${ }^{[9 \sim 11]}$. 因此, 在本组之前的研 究基础上, 我们设计合成了一系列具有不同卤素和邻位 三氟甲磺酸酯基取代的二芳基碘盐化合物, 并且通过核 磁共振和质谱进行结构表征. 在抗菌测试方面, 大肠杆 菌、金黄色葡萄球菌和枯草芽孢杆菌是常见的革兰氏阴 性菌和革兰氏阳性菌, 其特殊的细胞壁组成结构使化合 物的杀菌性存在差异 ${ }^{[12]}$. 根据 2014 年国家卫生部发布 的《消毒产品卫生安全评价规定》中对抗(抑)菌剂测试 项目的规定, 我们选用大肠杆菌、金黄色葡萄球菌和枯 草芽狍杆菌对目标化合物的抑菌性能进行测试, 研究了 卤素和邻位三氟甲磺酸酯基取代的二芳基碘盐对细菌 生长的影响, 并与市售卡松进行了比较. 另一方面, 我 们以最低抑菌浓度最低的化合物为目标抑菌剂, 对目标 化合物作用于大肠杆菌和金黄色葡萄球菌进行扫描电 子显微镜 (SEM) 测试; 对目标化合物与卡松水溶液对 Hela 细胞体外生长情况的影响进行了初步探究.

\section{1 结果与讨论}

\section{1 目标化合物的合成}

参照文献的合成方法 ${ }^{[2]}$, 目标化合物 $1 \sim 13$ 的合成 路线如 Scheme 1 所示. 利用不同卤素取代基的邻碘苯
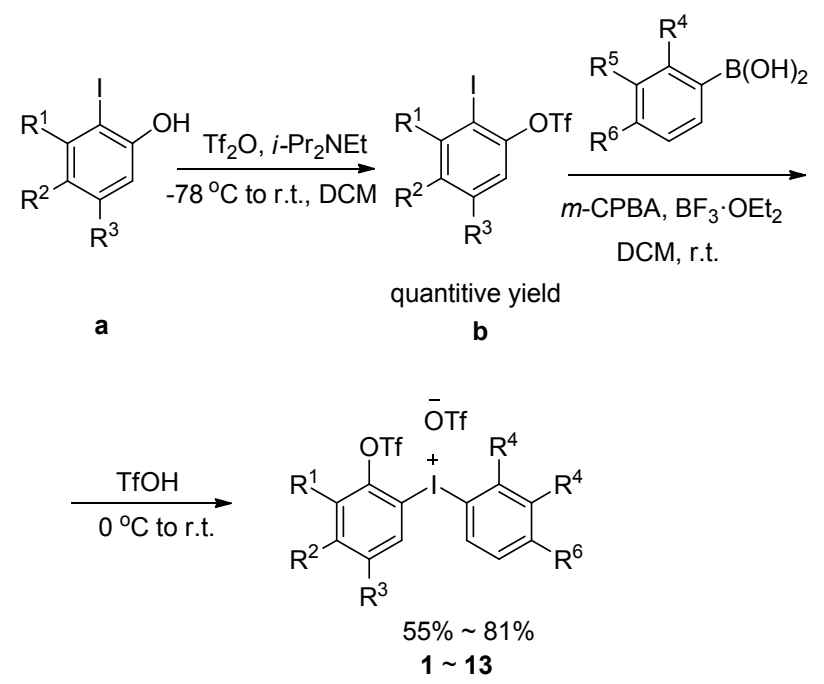

图式 1 目标化合物合成路线

Scheme 1 Synthetic route of target compound

酚 (a) 作为原料, 在低温 $-78{ }^{\circ} \mathrm{C}$ 下, 利用三氟甲磺酸䣶 和二异丙基乙基胺(DIPEA)保护酚羟基，以接近定量的 收率得到 2-碘苯三氟甲磺酸酯(b). 2-碘苯三氟甲磺酸酯 与具有不同取代基的苯嗍酸在间氯过氧苯甲酸氧化剂 和三氟化硼作为促进剂的共同作用下发生反应，无需分 离, 在低温下加入三氟甲磺酸成盐,一锅法生成一系列 具有不同卤素和邻位三氟甲磺酸酯基取代的二芳基碘 盐化合物 1 13. 经过乙醚重结晶得到目标产物, 收率 为 $55 \% \sim 81 \%$. 化合物 $\mathbf{1} \sim \mathbf{1 3}$ 的具体化学结构和反应收 率如图 1 所述.

\section{2 最低抑菌浓度测试}

以市售卡松 CIT/MIT 为对照品, 采用微量肉汤稀释 法 ${ }^{[13]}$ 测试化合物 1 13 对大肠杆菌、金黄色葡萄球菌和 枯草芽狍杆菌的最低抑菌浓度, 结果见表 1 所示(每个 化合物的抑菌曲线见辅助材料). 芳基上单卤素取代的 化合物 $1 \sim 9$ 的最低抑制浓度(MIC) 值为 $4 \sim 32 \mu \mathrm{g} / \mathrm{mL}$, 强于市售卡松的抗菌性能; 而芳基多卤素取代的化合物 10 和 13 的 MIC 值为 $16 \sim 64 \mu \mathrm{g} / \mathrm{mL}$, 抑菌效果好于卡 松或者与卡松抑菌效果相当. 值得高兴的是, 化合物 1 对大肠杆菌、金黄色葡萄球菌和枯草芽狍杆菌的 MIC 值分别为 $16,4,4 \mu \mathrm{g} / \mathrm{mL}$, 具有最好的抑菌活性. 化合 物 12 和 13 对金黄色葡萄球菌的抑制性能较差.

从以上结果可以看出, 苯环上不同位置具有不同卤 素单取代基的二芳基碘盐化合物对大肠杆菌、金黄色葡 萄球菌和枯草芽狍杆菌均具有一定的抑菌性. 其中, 取 代基为氟原子的化合物抑菌性能最强. 当作用于大肠杆 菌时, 单氟取代的化合物 1 的最低抑菌浓度约为取代基 为氯原子和澳原子化合物的 $1 / 2$ (表 1,1 对比 4 和 7); 另 外, 当化合物 12 与 13 对比, 结构仅相差一个氯原子和 
<smiles>Fc1cccc([In](c2ccccc2)c2ccccc2)c1</smiles><smiles>Fc1ccc(I(Br)c2ccccc2Br)cc1</smiles><smiles>COc1ccccc1[In][IH]c1ccccc1Cl</smiles><smiles>[13CH3]Oc1ccccc1I(Br)c1cccc(Cl)c1</smiles>

2, $80 \%$ yield<smiles>Cc1ccc(I(Br)c2ccccc2Br)cc1</smiles>

3, $73 \%$ yield<smiles>Brc1ccccc1[In][IH]c1ccccc1Br</smiles>

4, $70 \%$ yield<smiles>Brc1cccc(I(Br)c2ccccc2Br)c1</smiles>

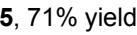

6, $65 \%$ yield<smiles>BrOc1ccccc1I(Br)c1ccc(Br)cc1</smiles><smiles>COc1ccc(F)cc1I(C)c1ccccc1</smiles>

8, $73 \%$ yield<smiles>Fc1cc(I(Br)c2ccccc2)c(O[Ga])c(F)c1F</smiles>

$11,55 \%$ yield<smiles>COc1c(F)cc(Cl)cc1I(Br)c1ccccc1</smiles>

$13,63 \%$ yield

图 1 目标化合物结构和产率

Figure 1 Chemical structures and yields of target compounds $1 \sim 13$

一个氟原子, 化合物 13 对金黄色葡萄球菌的 MIC 值明 显降低(表 1,12 对比 13). 然而奇怪的是, 当改变氟原子 取代基数量时, 如化合物 $\mathbf{9} \sim \mathbf{1 1}$, 随着氟原子的数目增 加, 化合物的抑菌性能呈现逐渐降低的趋势. 由于氟原 子的强吸电子性质, 抑菌活性与取代基的吸电子性能可 能存在一种平衡关系，当吸电子基数目增多至一定数目 时, 抑菌活性反而降低. 此外, 在卤素取代基的位置方 面，无论是氟、氯或是溴基取代，取代基的不同位置对 抑菌性的影响甚微(表 1, 1 对比 2,3 对比 4 和 5,6 对比 7 和 8 ).

\section{3 化合物 1 对细菌生长的影响}

以抑菌效果最优的化合物 1 为目标抑菌剂, 通过扫 描电子显微镜 $(\mathrm{SEM})$ 进一步探究化合物 1 对大肠杆菌 $(E$. coli $)$ 和金黄色葡萄球菌 $(S$. aureus $)$ 的杀菌活性差异. E. coli 与 $S$. aureus 分别与化合物 $1(128 \mu \mathrm{g} / \mathrm{mL})$ 于 $37{ }^{\circ} \mathrm{C}$, $200 \mathrm{r} / \mathrm{min}$ 培养 $7 \mathrm{~h}$ 后, SEM 结果见图 2 和 3 所示. 从结 果可以看出, $7 \mathrm{~h}$ 内化合物 1 对大肠杆菌和金黄色葡萄球
表 1 化合物 1 13 与卡松对大肠杆菌、金黄色葡萄球菌和枯 草芽孢杆菌的 MIC 值

Table 1 MIC values of compounds $\mathbf{1} \sim \mathbf{1 3}$ and Kathon to Escherichia coli, Bacillus subtilis and Staphylococcus aureus

\begin{tabular}{cccc}
\hline \multirow{2}{*}{ Compd. } & \multicolumn{3}{c}{$\mathrm{MIC} /\left(\mu \mathrm{g} \bullet \mathrm{mL}^{-1}\right)$} \\
\cline { 2 - 4 } & 大肠杆菌 & 金黄色葡萄球菌 & 枯草芽孢杆菌 \\
\hline $\mathbf{1}$ & 16 & 4 & 4 \\
$\mathbf{2}$ & 16 & 8 & 4 \\
$\mathbf{3}$ & 32 & 8 & 8 \\
$\mathbf{4}$ & 32 & 4 & 8 \\
$\mathbf{5}$ & 32 & 8 & 8 \\
$\mathbf{6}$ & 32 & 8 & 16 \\
$\mathbf{7}$ & 32 & 8 & 16 \\
$\mathbf{8}$ & 32 & 8 & 16 \\
$\mathbf{9}$ & 32 & 8 & 8 \\
$\mathbf{1 0}$ & 64 & 32 & 16 \\
$\mathbf{1 1}$ & 64 & $>128$ & 32 \\
$\mathbf{1 2}$ & 32 & $>128$ & 32 \\
$\mathbf{1 3}$ & 32 & 64 & 32 \\
$\mathrm{CIT} / \mathrm{MIT}^{a}$ & 64 & 64 & 32 \\
\hline
\end{tabular}

${ }^{a}$ CIT/MIT $=$ Commercial Kathon.

菌均产生作用, 细菌形态发生明显变化. 对比图 2-C 与 图 3-C, 在相同时间内，化合物 1 对金黄色葡萄球菌作 用效果更强，细菌形态裂解明显，抑菌活性更高. 此外, 对比 E. coli 与 S. aureus 的形态变化, 我们发现, 化合物 1 作用于细菌表面, 使细胞膜发生皱缩后破裂. 这一现 象表明，化合物 1 作用于大肠杆菌和金黄色葡萄球的差 异可能与细菌表面的结构不同存在一定关系，为进一步 揭示化合物 1 的抑菌机理提供了一个思路.
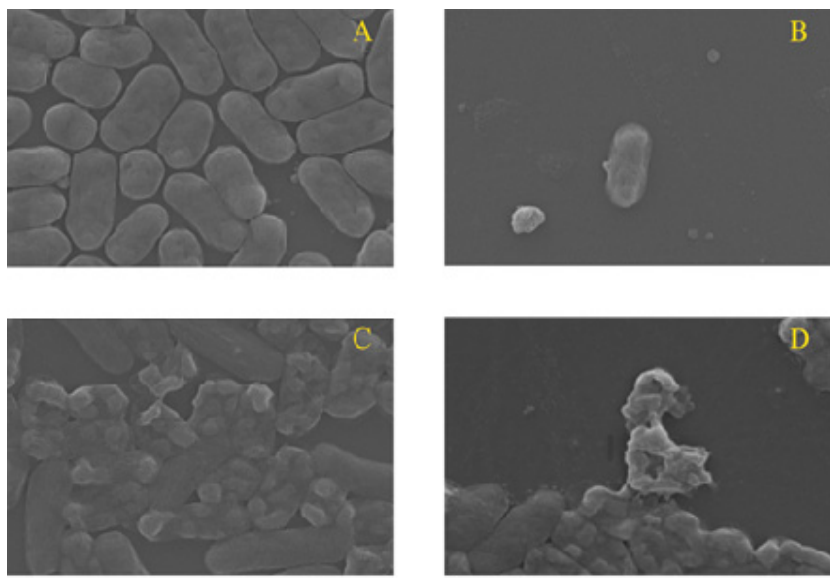

图 2 化合物 1 对大肠杆菌作用的 SEM 图像

Figure 2 SEM images of Effect of compound 1 on E. coli $\mathrm{A}$ and $\mathrm{B}$ are blank control, $\mathrm{C}$ and $\mathrm{D}$ are treatment of E. coli with compound 1 after $7 \mathrm{~h}$

\section{4 化合物 1 与卡松对 Hela 细胞生长的影响}

大多数抑菌剂均是通过诱导细胞调亡的方式发挥 细胞毒性作用. 在本研究中, 我们以抑菌性能最优的化 合物 1 与市售的卡松对细胞毒性进行测试. 通过显微 

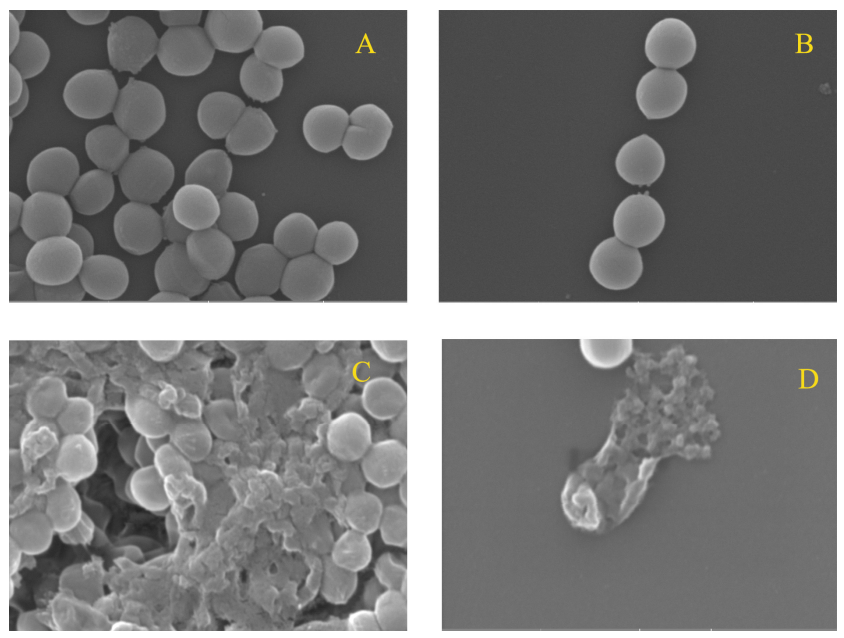

图 3 化合物 1 对金黄色葡萄球菌作用的 SEM 图像

Figure 3 SEM images of effect of compound 1 on S. aureus $\mathrm{A}$ and B are blank control, C and D are treatment of S. aureus with compound 1 after $7 \mathrm{~h}$

成像技术定性分析两种化合物对 Hela 细胞生长的影响, 测试结果见图 4 所示. Hela 细胞分别与化合物 1 (64 和 $128 \mu \mathrm{g} / \mathrm{mL})$ 和卡松 $(80$ 和 $160 \mu \mathrm{g} / \mathrm{mL})$ 共同卯育䏍 24 和 $48 \mathrm{~h}$ 后进行显微观察. 从显微成像技术测试结果可以看出, 化合物 1 和卡松水溶液在与细胞共同餒育 24 和 $48 \mathrm{~h}$ 后, 对 Hela 细胞生长的影响无明显差异.

为进一步定量地研究化合物 $\mathbf{1}$ 与卡松水溶液对 Hela 细胞的生长影响, 在不同浓度下, 采用噻唑蓝(MTT) 法 ${ }^{[14]}$ 对 Hela 细胞的体外细胞毒性进行测试, 对比结果 见图 5 所示. 相同浓度的 Hela 细胞分别与相同浓度的化 合物 1 和卡松水溶液共同捊育 $48 \mathrm{~h}$ 后, Hela 细胞的体外
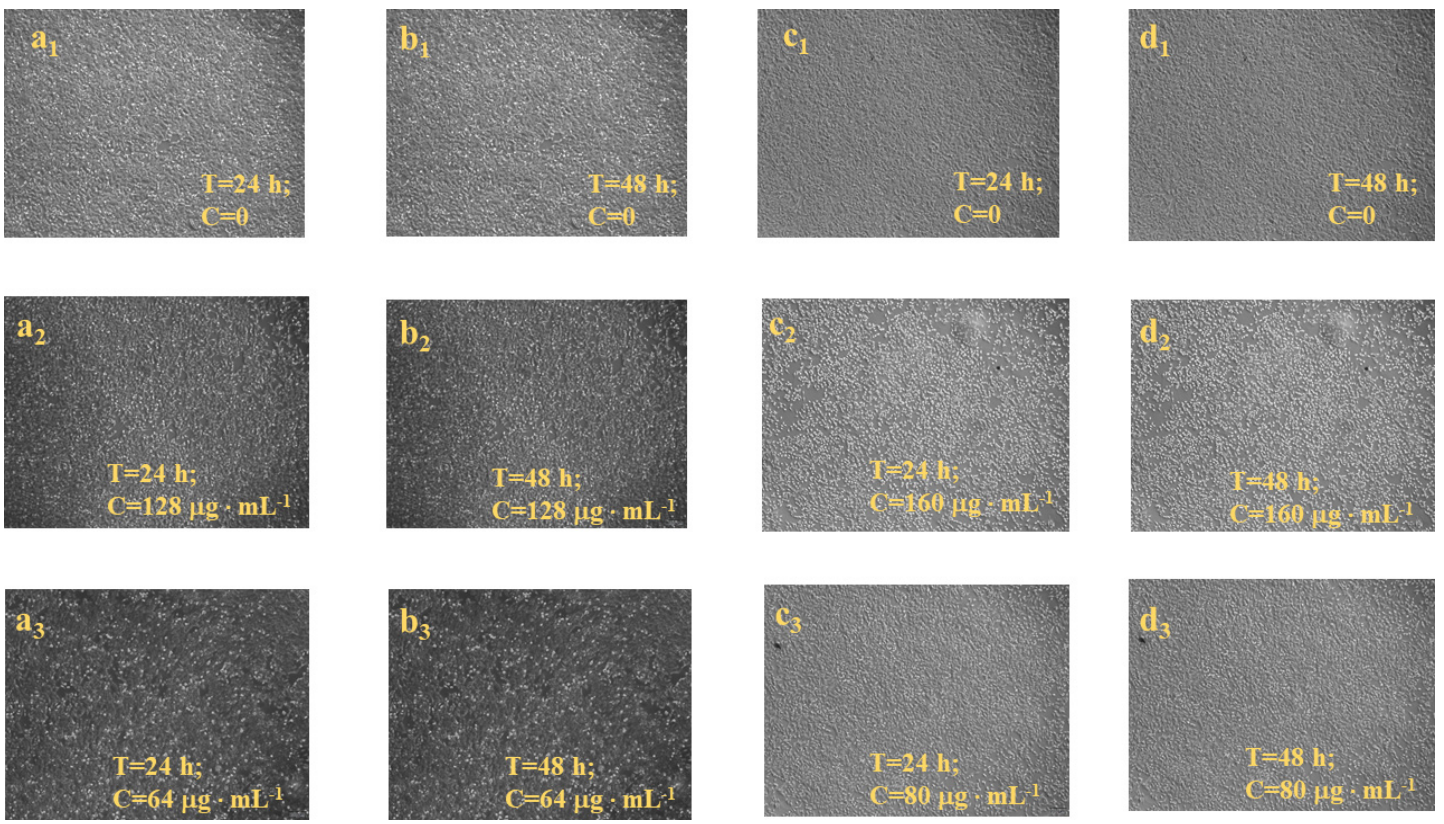

图 4 化合物 1 和卡松对 Hela 细胞生长的影响

Figure 4 Effects of compound 1 and kathon on Hela cell growth $a_{1} \sim a_{3}$ : compound 1 for $24 \mathrm{~h} ; b_{1} \sim b_{3}$ : compound 1 for $48 \mathrm{~h} ; c_{1} \sim c_{3}$ : Kathon for $24 \mathrm{~h} ; d_{1} \sim d_{3}$ : Kathon for $48 \mathrm{~h}$

存活率无较大差异, 化合物 1 与卡松对 Hela 细胞表现出 相当的体外细胞毒性. 显微成像技术和 MTT 法结果显 示在体外试验中, 化合物 1 对细胞造成的毒性与卡松水 溶液相当, 是一种低毒性的抗菌剂.

\section{2 结论}

合成了 13 种邻位三氟甲磺酸酯基和不同卤素多取 代的二芳基碘盐，并测试了对大肠杆菌、金黄色葡萄球 菌和枯草芽狍杆菌的最低抑菌浓度. 结果表明, 含有单 卤素取代基的化合物均具有优于市售抗菌剂卡松的抑 菌性能. 其中, 含有氟原子取代基的化合物性能更为显 著. 以抑菌性能最优的化合物 $\mathbf{1}$ 为目标抑菌剂, SEM 结 果表明, $7 \mathrm{~h}$ 内化合物对金黄色葡萄球菌和大肠杆菌的 形态改变较大, 具有明显的抑菌作用. 显微成像技术和 MTT 法细胞体外毒活性测试结果表明，与市售卡松比 较, 化合物 1 对 Hela 细胞体外生长影响与卡松相当, 是 一种低毒性的抑菌剂。该研究丰富了工业抑菌剂的种 类, 为减少微生物对工业抑菌剂的耐药性提供了一种可 选的新方案.

\section{3 实验部分}

\section{1 仪器与试剂}

核磁共振波谱仪(Bruker AM $400 \mathrm{MHz}$ )，电喷雾高 分辨质谱仪(Water LCT Premier XE), 台式离心机 (TGL-16C), 二氧化碳培养箱(QP-80), 酶标仪(Elx-808), 全温生化培养箱(SHP-150), 全温振荡器(HZP-150), 扫
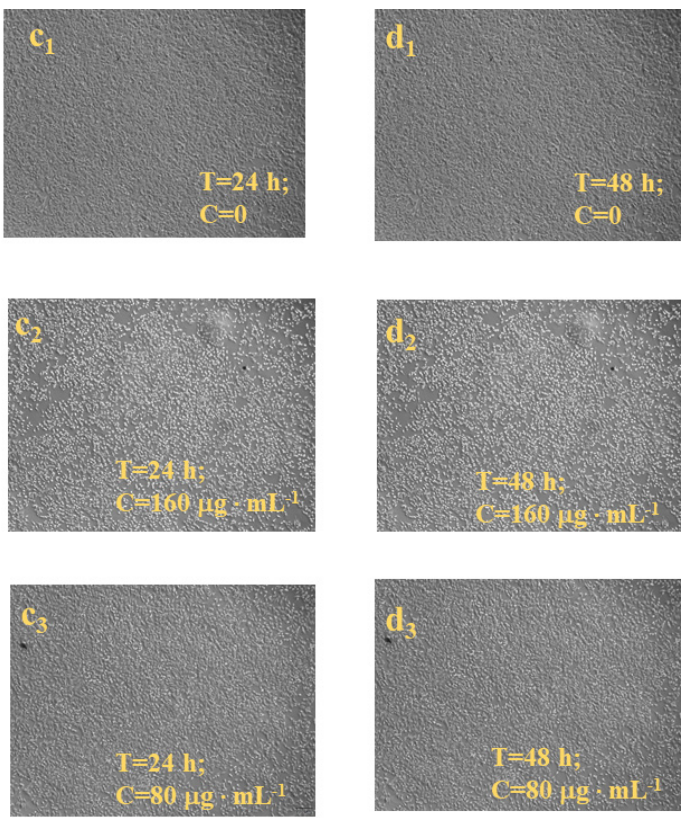


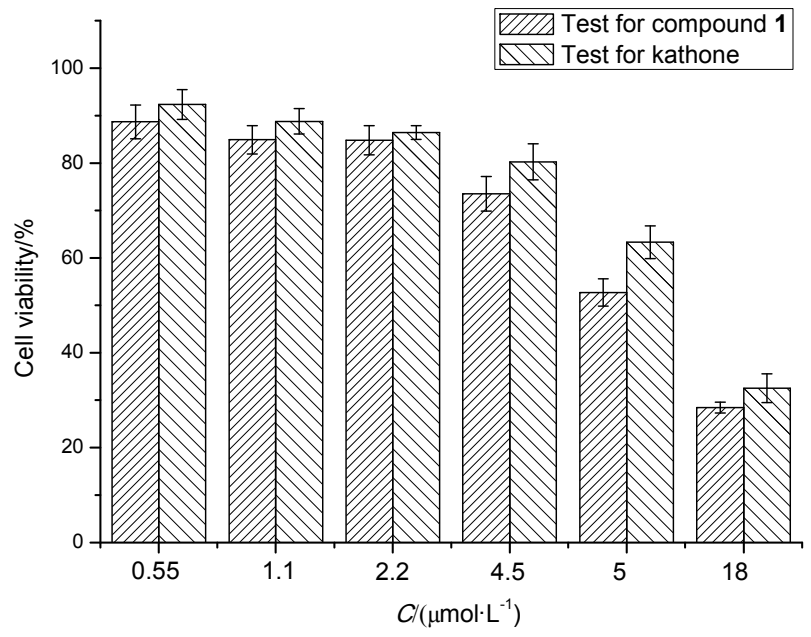

图 5 不同浓度的化合物 $\mathbf{1}$ 和卡松作用下 Hela 细胞的存活率 Figure 5 Survival rate of Hela cells with compound $\mathbf{1}$ or kathon in different concentrations

描电子显微镜(JEOL JSM-6360 LV), 紫外可见分光光度 计 752, 倒置显微镜 (XDS-900). 金黄色葡萄球菌 (ATCC25923), 大肠杆菌(ATCC25922), 枯草芽狍杆菌 (ATCC6633). 所有试剂与溶剂均为市售分析纯.

\section{2 化合物 1 13 的合成}

合成方法同参考文献[2]的制备方法. 在氮气氛中, $100 \mathrm{~mL}$ 的 Schlenk 反应瓶中依次加入邻碘苯基三氟甲磺 酸酯 $(5.0 \mathrm{mmol})$ 、间氯过氧苯甲酸 $(5.5 \mathrm{mmol})$ 和 $40 \mathrm{~mL}$ 二 氯甲烷溶剂. 在室温条件下, 向反应瓶中加入 2.5 equiv. 的三氟化嗍的乙醚溶液, 反应溶液变为黄色, 摚拌反应 $1 \mathrm{~h}$, 然后加入苯硼酸衍生物 $(5.5 \mathrm{mmol})$, 再搅拌反应 40 $\min$. 然后反应用冰水浴冷却至 $0{ }^{\circ} \mathrm{C}$, 滴加三氟甲磺酸 (6 mmol), 滴加完毕后撤掉冰水浴, 缓慢升至室温, 摚 拌 $15 \mathrm{~min}$; 反应完毕减压去除溶剂得到粗产物, 加入乙 醚重结晶得到目标产物 1 13.

(3-氟苯基)(2-(( 三氟甲基)磺酰基)氧基)苯基)三氟 甲磺酸碘盐(1): 白色粉末, $2.24 \mathrm{~g}$, 收率 75\%. m.p. 146 $148{ }^{\circ} \mathrm{C} ;{ }^{1} \mathrm{H}$ NMR (400 MHz, DMSO- $\left.d_{6}\right) \delta: 8.64$ (dd, $J=$ 8.0, $1.3 \mathrm{~Hz}, 1 \mathrm{H}), 8.20 \sim 8.15(\mathrm{~m}, 1 \mathrm{H}), 7.97(\mathrm{~d}, J=8.0 \mathrm{~Hz}$, $1 \mathrm{H}), 7.92 \sim 7.86(\mathrm{~m}, 1 \mathrm{H}), 7.78 \sim 7.74(\mathrm{~m}, 1 \mathrm{H}), 7.71 \sim 7.62$ $(\mathrm{m}, 2 \mathrm{H}), 7.58(\mathrm{td}, J=8.6,2.4 \mathrm{~Hz}, 1 \mathrm{H}) ;{ }^{13} \mathrm{C}$ NMR $(101$ MHz, DMSO- $\left.d_{6}\right) \delta: 161.90\left(\mathrm{~d}, J_{\mathrm{C}-\mathrm{F}}=253.5 \mathrm{~Hz}\right), 146.93$, $138.63,135.83,133.83\left(\mathrm{~d}, J_{\mathrm{C}-\mathrm{F}}=8.1 \mathrm{~Hz}\right), 131.86,131.31$ $\left(\mathrm{d}, J_{\mathrm{C}-\mathrm{F}}=3.0 \mathrm{~Hz}\right), 122.71,122.12\left(\mathrm{~d}, J_{\mathrm{C}-\mathrm{F}}=25.3 \mathrm{~Hz}\right)$, $120.70\left(\mathrm{q}, J_{\mathrm{C}-\mathrm{F}}=323.2 \mathrm{~Hz}\right), 120.01\left(\mathrm{~d}, J_{\mathrm{C}-\mathrm{F}}=20.2 \mathrm{~Hz}\right)$, $118.04\left(\mathrm{q}, J_{\mathrm{C}-\mathrm{F}}=322.2 \mathrm{~Hz}\right), 116.36\left(\mathrm{~d}, J_{\mathrm{C}-\mathrm{F}}=8.1 \mathrm{~Hz}\right)$, $111.13 ;{ }^{19} \mathrm{~F}$ NMR (376 MHz, DMSO- $\left.d_{6}\right) \delta:-72.97$ (s), -77.78 (s), -107.05 (s). HRMS calcd for $\mathrm{C}_{13} \mathrm{H}_{8} \mathrm{~F}_{4} \mathrm{IO}_{3} \mathrm{~S}$ $[\mathrm{M}-\mathrm{OTf}]^{+}$446.9175, found 446.9176.

(4-氟苯基)(2-(((三氟甲基)磺酰基)氧基)苯基)三氟
甲磺酸碘盐(2): 白色粉末, $2.38 \mathrm{~g}$, 收率 80\%. m.p. 176 $178{ }^{\circ} \mathrm{C} ;{ }^{1} \mathrm{H}$ NMR (400 MHz, DMSO- $\left.d_{6}\right) \delta: 8.65$ (d, $J=8.0$ $\mathrm{Hz}, 1 \mathrm{H}), 8.35 \sim 8.08(\mathrm{~m}, 2 \mathrm{H}), 7.95 \sim 7.81(\mathrm{~m}, 1 \mathrm{H}), 7.74(\mathrm{~d}$, $J=8.3 \mathrm{~Hz}, 1 \mathrm{H}), 7.71 \sim 7.62(\mathrm{~m}, 1 \mathrm{H}), 7.45(\mathrm{t}, J=8.8 \mathrm{~Hz}$, $2 \mathrm{H}) ;{ }^{13} \mathrm{C}$ NMR $\left(101 \mathrm{MHz}\right.$, DMSO- $\left.d_{6}\right) \delta: 164.16\left(\mathrm{~d}, J_{\mathrm{C}-\mathrm{F}}=\right.$ $253.2 \mathrm{~Hz}), 146.96,138.59,137.95\left(\mathrm{~d}, J_{\mathrm{C}-\mathrm{F}}=9.3 \mathrm{~Hz}\right)$, $136.58\left(\mathrm{~d}, J_{\mathrm{C}-\mathrm{F}}=8.0 \mathrm{~Hz}\right), 135.74,131.83,122.72,120.76$ $\left(\mathrm{q}, J_{\mathrm{C}-\mathrm{F}}=323.2 \mathrm{~Hz}\right), 119.63\left(\mathrm{~d}, J_{\mathrm{C}-\mathrm{F}}=23.1 \mathrm{~Hz}\right), 118.09(\mathrm{q}$, $\left.J_{\mathrm{C}-\mathrm{F}}=322.2 \mathrm{~Hz}\right), 111.27\left(\mathrm{~d}, J_{\mathrm{C}-\mathrm{F}}=3.0 \mathrm{~Hz}\right) ;{ }^{19} \mathrm{~F}$ NMR $(376$ MHz, DMSO- $\left.d_{6}\right) \delta$ : $-73.06(\mathrm{~s}),-77.82(\mathrm{~s}),-105.84$ (s). HRMS calcd for $\mathrm{C}_{13} \mathrm{H}_{8} \mathrm{~F}_{4} \mathrm{IO}_{3} \mathrm{~S}$ [M-OTf] $]^{+} 446.9175$, found 446.9177.

(2-氯苯基)(2-(((三氟甲基)磺酰基)氧基)苯基)三氟 甲磺酸碘盐(3): 白色粉末, $2.19 \mathrm{~g}$, 收率 73\%. m.p. 173 $174{ }^{\circ} \mathrm{C} ;{ }^{1} \mathrm{H}$ NMR $\left(400 \mathrm{MHz}\right.$, DMSO- $\left.d_{6}\right) \delta: 8.64$ (d, $J=8.0$ $\mathrm{Hz}, 1 \mathrm{H}), 8.47(\mathrm{~d}, J=8.0 \mathrm{~Hz}, 1 \mathrm{H}), 7.91 \sim 7.82(\mathrm{~m}, 2 \mathrm{H})$, $7.76 \sim 7.69(\mathrm{~m}, 2 \mathrm{H}), 7.65(\mathrm{td}, J=7.9,1.2 \mathrm{~Hz}, 1 \mathrm{H}), 7.53(\mathrm{td}$, $J=7.9,1.4 \mathrm{~Hz}, 1 \mathrm{H}) ;{ }^{13} \mathrm{C}$ NMR $\left(101 \mathrm{MHz}\right.$, DMSO- $\left.d_{6}\right) \delta$ : $147.19,138.71,138.51,135.77,135.67,135.07,131.57$, $130.66,130.50,120.73\left(\mathrm{q}, J_{\mathrm{C}-\mathrm{F}}=323.2 \mathrm{~Hz}\right), 122.22$, $120.02,118.07\left(\mathrm{q}, J_{\mathrm{C}-\mathrm{F}}=322.2 \mathrm{~Hz}\right), 110.86 ;{ }^{19} \mathrm{~F}$ NMR $(376$ MHz, DMSO- $\left.d_{6}\right) \delta:-73.26(\mathrm{~s}),-77.79$ (s). HRMS calcd for $\mathrm{C}_{13} \mathrm{H}_{8} \mathrm{~F}_{3} \mathrm{ClIO}_{3} \mathrm{~S}[\mathrm{M}-\mathrm{OTf}]^{+}$462.8880, found 462.8879 .

(3-氯苯基)(2-(((三氟甲基)磺酰基)氧基)苯基)三氟 甲磺酸碘盐(4): 白色粉末, $2.14 \mathrm{~g}$, 收率 70\%. m.p. 140 $141{ }^{\circ} \mathrm{C} ;{ }^{1} \mathrm{H}$ NMR (400 MHz, DMSO- $\left.d_{6}\right) \delta:: 8.74 \sim 8.62$ (m, $1 \mathrm{H}), 8.34$ (t, $J=1.8 \mathrm{~Hz}, 1 \mathrm{H}), 8.10$ (d, $J=8.2 \mathrm{~Hz}, 1 \mathrm{H})$, $7.96 \sim 7.82(\mathrm{~m}, 1 \mathrm{H}), 7.76(\mathrm{t}, J=7.1 \mathrm{~Hz}, 2 \mathrm{H}), 7.73 \sim 7.66$ (m, 1H), $7.61(\mathrm{t}, J=8.1 \mathrm{~Hz}, 1 \mathrm{H}) ;{ }^{13} \mathrm{C}$ NMR $(101 \mathrm{MHz}$, DMSO- $\left.d_{6}\right) \delta: 147.00,138.71,135.87,135.19,134.19$, $133.64,133.51,132.71,131.90,122.73,120.75$ (q, $J_{\mathrm{C}-\mathrm{F}}=$ $323.2 \mathrm{~Hz}), 118.08\left(\mathrm{q}, J_{\mathrm{C}-\mathrm{F}}=322.2 \mathrm{~Hz}\right), 117.18,111.16 ;{ }^{19} \mathrm{~F}$ NMR (376 MHz, DMSO- $\left.d_{6}\right) \delta$ : $-73.06(\mathrm{~s}),-77.82(\mathrm{~s})$. HRMS calcd for $\mathrm{C}_{13} \mathrm{H}_{8} \mathrm{~F}_{3} \mathrm{ClIO}_{3} \mathrm{~S}[\mathrm{M}-\mathrm{OTf}]^{+} 462.8880$, found 462.8873 .

(4-氯苯基)(2-(( 三氟甲基)磺酰基)氧基)苯基)三氟 甲磺酸碘盐(5): 白色粉末, $2.17 \mathrm{~g}$, 收率 71\%. m.p. 186 $188{ }^{\circ} \mathrm{C} ;{ }^{1} \mathrm{H}$ NMR (400 MHz, DMSO- $\left.d_{6}\right) \delta: 8.64$ (dd, $J=$ $8.0,1.5 \mathrm{~Hz}, 1 \mathrm{H}), 8.22 \sim 8.07(\mathrm{~m}, 2 \mathrm{H}), 7.95 \sim 7.83(\mathrm{~m}, 1 \mathrm{H})$, $7.81 \sim 7.73(\mathrm{~m}, 1 \mathrm{H}), 7.72 \sim 7.60(\mathrm{~m}, 3 \mathrm{H}) ;{ }^{13} \mathrm{C}$ NMR $(101$ $\left.\mathrm{MHz}, \mathrm{DMSO}-d_{6}\right) \delta: 146.95,138.62,136.77,136.00$, $135.79,132.12,127.48,122.75,120.72$ (q, $J_{\mathrm{C}-\mathrm{F}}=323.2$ $\mathrm{Hz}), 118.05\left(\mathrm{q}, J_{\mathrm{C}-\mathrm{F}}=321.2 \mathrm{~Hz}\right), 114.85,111.18 ;{ }^{19} \mathrm{~F}$ NMR $\left(376 \mathrm{MHz}, \mathrm{DMSO}-d_{6}\right) \delta$ : $-73.00(\mathrm{~s}),-77.80$ (s). HRMS 
calcd for $\mathrm{C}_{13} \mathrm{H}_{8} \mathrm{~F}_{3} \mathrm{ClIO}_{3} \mathrm{~S}[\mathrm{M}-\mathrm{OTf}]^{+}$462.8880, found 462.8884 .

(2-溴苯基)(2-(((三氟甲基)磺酰基)氧基)苯基)三氟 甲磺酸碘盐(6): 白色粉末, $2.13 \mathrm{~g}$, 收率 65\%. m.p. 202 $204{ }^{\circ} \mathrm{C} ;{ }^{1} \mathrm{H}$ NMR $\left(400 \mathrm{MHz}\right.$, DMSO- $\left.d_{6}\right) \delta: 8.61$ (d, $J=7.9$ $\mathrm{Hz}, 1 \mathrm{H}), 8.44(\mathrm{~d}, J=7.8 \mathrm{~Hz}, 1 \mathrm{H}), 7.99(\mathrm{dd}, J=7.9,1.4 \mathrm{~Hz}$, $1 \mathrm{H}), 7.93 \sim 7.82(\mathrm{~m}, 1 \mathrm{H}), 7.74(\mathrm{dd}, J=8.3,1.0 \mathrm{~Hz}, 1 \mathrm{H})$, 7.64 (ddd, $J=9.4,8.1,1.4 \mathrm{~Hz}, 2 \mathrm{H}), 7.57$ (td, $J=7.7,1.5$ $\mathrm{Hz}, 1 \mathrm{H}) ;{ }^{13} \mathrm{C}$ NMR $\left(101 \mathrm{MHz}\right.$, DMSO- $\left.d_{6}\right) \delta: 147.24$, $138.83,138.64,135.79,135.01,134.06,131.55,130.92$, $126.54,123.07,122.32,120.75$ (q, $\left.J_{\mathrm{C}-\mathrm{F}}=331.3 \mathrm{~Hz}\right), 118.04$ $\left(\mathrm{q}, J_{\mathrm{C}-\mathrm{F}}=322.2 \mathrm{~Hz}\right), 111.07 ;{ }^{19} \mathrm{~F}$ NMR $(376 \mathrm{MHz}$, DMSO- $\left.d_{6}\right) \delta$ : $-73.17(\mathrm{~s}),-77.79$ (s). HRMS calcd for $\mathrm{C}_{13} \mathrm{H}_{8} \mathrm{~F}_{3} \mathrm{BrIO}_{3} \mathrm{~S}[\mathrm{M}-\mathrm{OTf}]^{+}$506.8374, found 506.8376.

(3-澳苯基)(2-(((三氟甲基)磺酰基)氧基)苯基)三氟 甲磺酸碘盐(7): 白色粉末, $2.07 \mathrm{~g}$, 收率 $69 \%$. m.p. $164 \sim 166{ }^{\circ} \mathrm{C} ;{ }^{1} \mathrm{H}$ NMR $\left(400 \mathrm{MHz}\right.$, DMSO- $\left.d_{6}\right) \delta: 8.65(\mathrm{dd}$, $J=8.0,1.2 \mathrm{~Hz}, 1 \mathrm{H}), 8.45$ (t, $J=1.7 \mathrm{~Hz}, 1 \mathrm{H}), 8.16 \sim 8.10$ (m, 1H), 7.89 (dd, $J=12.2,5.2 \mathrm{~Hz}, 2 \mathrm{H}), 7.78 \sim 7.73(\mathrm{~m}$, 1H), 7.70 (td, $J=7.9,1.2 \mathrm{~Hz}, 1 \mathrm{H}), 7.54(\mathrm{t}, J=8.1 \mathrm{~Hz}, 1 \mathrm{H})$; ${ }^{13} \mathrm{C}$ NMR (101 MHz, DMSO- $\left.d_{6}\right) \quad \delta$ : 146.96, 138.67, $136.73,135.86,135.51,133.87,133.82,131.88,123.49$, $122.72,120.70\left(\mathrm{q}, J_{\mathrm{C}-\mathrm{F}}=324.2 \mathrm{~Hz}\right), 118.05\left(\mathrm{q}, J_{\mathrm{C}-\mathrm{F}}=322.2\right.$ $\mathrm{Hz}), 117.47,111.12 ;{ }^{19} \mathrm{~F}$ NMR (376 MHz, DMSO- $\left.d_{6}\right) \delta$ : -72.99 (s), -77.77 (s). HRMS calcd for $\mathrm{C}_{13} \mathrm{H}_{8} \mathrm{~F}_{3} \mathrm{BrIO}_{3} \mathrm{~S}$ $[\mathrm{M}-\mathrm{OTf}]^{+}$506.8374, found 506.8374.

(4-溴苯基)(2-(((三氟甲基)磺酰基)氧基)苯基)三氟 甲磺酸碘盐 $(8)$ : 白色粉末, $2.4 \mathrm{~g}$, 收率 73\%. m.p. 162 $164{ }^{\circ} \mathrm{C} ;{ }^{1} \mathrm{H}$ NMR (400 MHz, DMSO- $\left.d_{6}\right) \delta: 8.63$ (dd, $J=$ 8.0, $1.5 \mathrm{~Hz}, 1 \mathrm{H}), 8.11 \sim 8.02(\mathrm{~m}, 2 \mathrm{H}), 7.93 \sim 7.85(\mathrm{~m}, 1 \mathrm{H})$, $7.82 \sim 7.78(\mathrm{~m}, 2 \mathrm{H}), 7.78 \sim 7.73(\mathrm{~m}, 1 \mathrm{H}), 7.68(\mathrm{td}, J=7.9$, $1.3 \mathrm{~Hz}, 1 \mathrm{H}) ;{ }^{13} \mathrm{C}$ NMR (101 MHz, DMSO-d $\left.d_{6}\right) \delta: 146.95$, $138.61,136.82,135.78,135.00,131.83,126.70,122.76$, $120.71\left(\mathrm{q}, J_{\mathrm{C}-\mathrm{F}}=323.5 \mathrm{~Hz}\right), 118.04\left(\mathrm{q}, J_{\mathrm{C}-\mathrm{F}}=321.9 \mathrm{~Hz}\right)$, 115.56, 111.12; ${ }^{19} \mathrm{~F}$ NMR (376 MHz, DMSO- $\left.d_{6}\right) \quad \delta$ : -72.98 (s), -77.78 (s). HRMS calcd for $\mathrm{C}_{13} \mathrm{H}_{8} \mathrm{~F}_{3} \mathrm{BrIO}_{3} \mathrm{~S}$ $[\mathrm{M}-\mathrm{OTf}]^{+}$506.8374, found 506.8387.

(5-氟-2-(((三氟甲基)磺酰基)氧基)苯基)(苯基)三氟 甲磺酸碘盐(9): 白色粉末, $2.11 \mathrm{~g}$, 收率 71\%. m.p. 148 $150{ }^{\circ} \mathrm{C} ;{ }^{1} \mathrm{H}$ NMR (400 MHz, DMSO- $\left.d_{6}\right) \delta: 8.70$ (dd, $J=$ $7.2,3.0 \mathrm{~Hz}, 1 \mathrm{H}), 8.19$ (d, $J=7.5 \mathrm{~Hz}, 2 \mathrm{H}), 7.84$ (dd, $J=9.2$, $4.3 \mathrm{~Hz}, 1 \mathrm{H}), 7.79 \sim 7.67(\mathrm{~m}, 2 \mathrm{H}), 7.60(\mathrm{t}, J=7.7 \mathrm{~Hz}, 2 \mathrm{H})$; ${ }^{13} \mathrm{C}$ NMR (101 MHz, DMSO- $\left.d_{6}\right) \delta: 160.71\left(\mathrm{~d}, J_{\mathrm{C}-\mathrm{F}}=255.2\right.$ $\mathrm{Hz}), 143.54\left(\mathrm{~d}, J_{\mathrm{C}-\mathrm{F}}=3.3 \mathrm{~Hz}\right), 135.03,132.73,132.26$, $125.38\left(\mathrm{~d}, J_{\mathrm{C}-\mathrm{F}}=27.3 \mathrm{~Hz}\right), 124.44\left(\mathrm{~d}, J_{\mathrm{C}-\mathrm{F}}=8.7 \mathrm{~Hz}\right)$, $122.32\left(\mathrm{~d}, J_{\mathrm{C}-\mathrm{F}}=24.1 \mathrm{~Hz}\right), 120.72\left(\mathrm{q}, J_{\mathrm{C}-\mathrm{F}}=323.5 \mathrm{~Hz}\right)$, $118.05\left(\mathrm{q}, J_{\mathrm{C}-\mathrm{F}}=321.8 \mathrm{~Hz}\right), 117.35,111.77\left(\mathrm{~d}, J_{\mathrm{C}-\mathrm{F}}=8.6\right.$ $\mathrm{Hz}) ;{ }^{19} \mathrm{~F}$ NMR (376 MHz, DMSO- $d_{6}$ ) $\delta$ : -72.97 (s), -77.82 (s), -107.70 (s). HRMS calcd for $\mathrm{C}_{13} \mathrm{H}_{8} \mathrm{~F}_{4} \mathrm{IO}_{3} \mathrm{~S}$ $[\mathrm{M}-\mathrm{OTf}]^{+} 446.9175$, found 446.9174 .

(4-(5-二氟-2-((三氟甲基)磺酰基)氧基)苯基)(苯基) 三氟甲磺酸碘盐(10): 白色粉末, $2.06 \mathrm{~g}$, 收率 67\%. m.p. $154 \sim 155{ }^{\circ} \mathrm{C} ;{ }^{1} \mathrm{H}$ NMR (400 MHz, DMSO- $\left.d_{6}\right) \delta: 8.95(\mathrm{t}$, $J=8.6 \mathrm{~Hz}, 1 \mathrm{H}), 8.18(\mathrm{~d}, J=7.9 \mathrm{~Hz}, 2 \mathrm{H}), 8.11(\mathrm{dd}, J=$ 10.0, $6.4 \mathrm{~Hz}, 1 \mathrm{H}), 7.71(\mathrm{t}, J=7.4 \mathrm{~Hz}, 1 \mathrm{H}), 7.59$ (dd, $J=$ 10.7, $4.8 \mathrm{~Hz}, 2 \mathrm{H}) .{ }^{13} \mathrm{C}$ NMR (101 MHz, DMSO- $\left.d_{6}\right) \delta$ : $152.21\left(\mathrm{dd}, J_{\mathrm{C}-\mathrm{F}}=259.9,13.6 \mathrm{~Hz}\right), 149.42\left(\mathrm{dd}, J_{\mathrm{C}-\mathrm{F}}=\right.$ 257.4, $12.9 \mathrm{~Hz}), 143.42\left(\mathrm{dd}, J_{\mathrm{C}-\mathrm{F}}=9.5,3.3 \mathrm{~Hz}\right), 135.00$, $132.73,132.26,126.68\left(\mathrm{~d}, J_{\mathrm{C}-\mathrm{F}}=21.9 \mathrm{~Hz}\right), 120.75\left(\mathrm{q}, J_{\mathrm{C}-\mathrm{F}}\right.$ $=323.5 \mathrm{~Hz}), 118.04\left(\mathrm{q}, J_{\mathrm{C}-\mathrm{F}}=322.1 \mathrm{~Hz}\right), 117.50,113.54$ $\left(\mathrm{d}, J_{\mathrm{C}-\mathrm{F}}=22.8 \mathrm{~Hz}\right), 106.04\left(\mathrm{t}, J_{\mathrm{C}-\mathrm{F}}=5.0 \mathrm{~Hz}\right) ;{ }^{19} \mathrm{~F}$ NMR $(376$ $\left.\mathrm{MHz}, \mathrm{DMSO}-d_{6}\right) \delta$ : $-72.73(\mathrm{~s}),-77.87(\mathrm{~s}),-123.90$ (d, $J=28.7 \mathrm{~Hz}),-131.17$ (d, $J=25.4 \mathrm{~Hz})$. HRMS calcd for $\mathrm{C}_{13} \mathrm{H}_{7} \mathrm{~F}_{5} \mathrm{IO}_{3} \mathrm{~S}[\mathrm{M}-\mathrm{OTf}]^{+}$464.9081, found 464.9084 .

(3,4,5-三氟-2-(( 三氟甲基)磺酰基)氧基)苯基)(苯 基)三氟甲磺酸碘盐(11): 黄色粉末, $1.74 \mathrm{~g}$, 收率 $55 \%$. m.p. $147 \sim 149{ }^{\circ} \mathrm{C} ;{ }^{1} \mathrm{H}$ NMR (400 MHz, DMSO- $d_{6}$ ) $\delta$ : 8.34 (td, $J=9.0,2.4 \mathrm{~Hz}, 1 \mathrm{H}), 8.07$ (d, $J=7.4 \mathrm{~Hz}, 2 \mathrm{H}), 7.58$ (t, $J=7.4 \mathrm{~Hz}, 1 \mathrm{H}), 7.46(\mathrm{t}, J=7.7 \mathrm{~Hz}, 2 \mathrm{H}) ;{ }^{13} \mathrm{C}$ NMR $(101$ MHz, DMSO- $\left.d_{6}\right) \delta: 142.77,142.65\left(\mathrm{~d}, J_{\mathrm{C}-\mathrm{F}}=3.0 \mathrm{~Hz}\right)$, $142.58,134.67,131.39,131.34,120.74\left(\mathrm{q}, J_{\mathrm{C}-\mathrm{F}}=323.2\right.$ $\mathrm{Hz}), 118.74\left(\mathrm{~d}, J_{\mathrm{C}-\mathrm{F}}=4.0 \mathrm{~Hz}\right), 118.52\left(\mathrm{~d}, J_{\mathrm{C}-\mathrm{F}}=3.0 \mathrm{~Hz}\right)$, $117.54\left(\mathrm{q}, J_{\mathrm{C}-\mathrm{F}}=324.2 \mathrm{~Hz}\right), 114.98,104.86 ;{ }^{19} \mathrm{~F}$ NMR $(376$ $\left.\mathrm{MHz}, \mathrm{DMSO}-d_{6}\right) \delta$ : $-72.93(\mathrm{~s}),-77.79(\mathrm{~s}),-144.45$ (d, $J=22.7 \mathrm{~Hz}),-149.45$ (d, $J=21.1 \mathrm{~Hz}),-151.96$ (t, $J=22.2 \mathrm{~Hz}$ ). HRMS calcd for $\mathrm{C}_{13} \mathrm{H}_{6} \mathrm{~F}_{6} \mathrm{IO}_{3} \mathrm{~S}[\mathrm{M}-\mathrm{OTf}]^{+}$ 482.8987, found 482.8994.

(3,5-二氯-2-(((三氟甲基)磺酰基)氧基)苯基)(苯基) 三氟甲磺酸碘盐(12): 白色粉末, $2.62 \mathrm{~g}$, 收率 $81 \%$. m.p. $170 \sim 172{ }^{\circ} \mathrm{C} ;{ }^{1} \mathrm{H}$ NMR $\left(400 \mathrm{MHz}, \mathrm{DMSO}-d_{6}\right) \delta: 8.84(\mathrm{~d}$, $J=2.4 \mathrm{~Hz}, 1 \mathrm{H}), 8.36 \sim 8.29(\mathrm{~m}, 1 \mathrm{H}), 8.19(\mathrm{~d}, J=7.7 \mathrm{~Hz}$, $2 \mathrm{H}), 7.72(\mathrm{t}, J=7.4 \mathrm{~Hz}, 1 \mathrm{H}), 7.61(\mathrm{t}, J=7.8 \mathrm{~Hz}, 2 \mathrm{H}) .{ }^{13} \mathrm{C}$ NMR (101 MHz, DMSO- $\left.d_{6}\right) \delta: 150.89,142.12,136.91$, $135.20,132.37,131.85,125.26,120.74\left(\mathrm{q}, J_{\mathrm{C}-\mathrm{F}}=323.2\right.$ $\mathrm{Hz}), 118.23\left(\mathrm{q}, J_{\mathrm{C}-\mathrm{F}}=323.2 \mathrm{~Hz}\right), 117.63,113.84,108.81$. ${ }^{19} \mathrm{~F}$ NMR $\left(376 \mathrm{MHz}\right.$, DMSO- $\left.d_{6}\right) \delta:-70.63(\mathrm{~s}),-77.79$ (s). HRMS calcd for $\mathrm{C}_{13} \mathrm{H}_{7} \mathrm{~F}_{3} \mathrm{Cl}_{2} \mathrm{IO}_{3} \mathrm{~S} \quad[\mathrm{M}-\mathrm{OTf}]^{+}$ 496.8490, found 496.8492 .

(5-氯-3-氟-2-(((三氟甲基)磺酰基)氧基)苯基)(苯基) 三氟甲磺酸碘盐(13): 白色粉末, $1.98 \mathrm{~g}$, 收率 63\%. m.p. 
$176 \sim 178{ }^{\circ} \mathrm{C} ;{ }^{1} \mathrm{H}$ NMR $\left(400 \mathrm{MHz}\right.$, DMSO- $\left.d_{6}\right) \delta: 8.73(\mathrm{t}$, $J=2.0 \mathrm{~Hz}, 1 \mathrm{H}), 8.27(\mathrm{dd}, J=10.0,2.3 \mathrm{~Hz}, 1 \mathrm{H}), 8.20(\mathrm{~d}$, $J=8.1 \mathrm{~Hz}, 2 \mathrm{H}), 7.73$ (t, $J=7.4 \mathrm{~Hz}, 1 \mathrm{H}), 7.61$ (t, $J=7.8$ $\mathrm{Hz}, 2 \mathrm{H}) ;{ }^{13} \mathrm{C}$ NMR (101 MHz, DMSO- $\left.d_{6}\right) \delta: 152.14(\mathrm{~d}$, $\left.J_{\mathrm{C}-\mathrm{F}}=260.6 \mathrm{~Hz}\right), 136.33\left(\mathrm{~d}, J_{\mathrm{C}-\mathrm{F}}=10.1 \mathrm{~Hz}\right), 135.09,134.26$ $\left(\mathrm{d}, J_{\mathrm{C}-\mathrm{F}}=15.1 \mathrm{~Hz}\right), 133.22\left(\mathrm{~d}, J_{\mathrm{C}-\mathrm{F}}=4.0 \mathrm{~Hz}\right), 132.89$, $132.40,123.49\left(\mathrm{~d}, J_{\mathrm{C}-\mathrm{F}}=23.2 \mathrm{~Hz}\right), 120.75\left(\mathrm{q}, J_{\mathrm{C}-\mathrm{F}}=323.2\right.$ $\mathrm{Hz}), 118.18$ (q, $\left.J_{\mathrm{C}-\mathrm{F}}=322.2 \mathrm{~Hz}\right), 117.64,113.66 ;{ }^{19} \mathrm{~F}$ NMR $\left(376 \mathrm{MHz}, \mathrm{DMSO}-d_{6}\right) \delta:-71.67(\mathrm{~s}),-77.80(\mathrm{~s})$, $-117.25\left(\mathrm{~d}, J=15.7 \mathrm{~Hz}\right.$ ). HRMS calcd for $\mathrm{C}_{13} \mathrm{H}_{7} \mathrm{~F}_{4} \mathrm{ClIO}_{3} \mathrm{~S}$ $[\mathrm{M}-\mathrm{OTf}]^{+} 480.8785$, found 480.8783 .

\subsection{MIC 值测试}

以市售卡松为对照组, 采用微量肉汤稀释法测定目 标化合物的最低抑菌浓度. 取已活化的对数生长期的细 菌 $\left(1 \times 10^{6} \mathrm{CFU} / \mathrm{mL}\right)$ 接种于 96 孔板中, 设置 12 个浓度 梯度, 每个浓度 3 个复孔, $37{ }^{\circ} \mathrm{C}, 200 \mathrm{r} / \mathrm{min}$ 培养 $24 \mathrm{~h}$ 后, 酶标仪 $600 \mathrm{~nm}$ 波长测定吸光度, 绘制浓度与吸光度曲 线得到 MIC 值.

\section{4 扫描电子显微镜测试}

取待测样品, $2000 \mathrm{r} / \mathrm{min}$ 离心 $12 \mathrm{~min}$ 后收集沉淀. $4{ }^{\circ} \mathrm{C}$ 下加入质量分数为 $2.5 \%$ 的戊二醛固定 $12 \mathrm{~h}$ 后加入 磷酸盐缓冲液洗涤 3 次. 设置 5 个浓度梯度, 依次增加 乙醇浓度使细胞脱水, $2000 \mathrm{r} / \mathrm{min}$ 离心 $12 \mathrm{~min}$. 沉淀物中 加入少量无水乙醇重悬后, 负载至玻璃上烘干, SEM 测 试并记录结果.

\section{5 显微成像技术}

采用显微成像技术定性测试化合物 $\mathbf{1}$ 与卡松水溶液 的体外细胞毒活性. 取对数生长期的 Hela 细胞 $\left(5 \times 10^{4}\right.$ 个 $/ \mathrm{mL}$ )接种于 12 孔板中, 过夜培养后加入待测化合物. 设置化合物 1 浓度为 64 和 $128 \mu \mathrm{g} / \mathrm{mL}$, 卡松水溶液浓度 为 80 和 $160 \mu \mathrm{g} / \mathrm{mL}$. 24 和 $48 \mathrm{~h}$ 后分别用显微镜观察并记 录细胞生长状态.

\subsection{MTT 细胞毒性测试}

采用 MTT 法定量测定化合物 $\mathbf{1}$ 与卡松的体外细胞 毒活性. 取对数生长期的 Hela 细胞 $\left(10^{4}\right.$ 个 $\left./ \mathrm{mL}\right)$ 接种于 96
孔板中, 过夜培养后加入待测化合物, 设置 6 个浓度梯 度, 每个浓度 3 个复孔. 作用 $48 \mathrm{~h}$ 后, 每孔加入 $10 \mu \mathrm{L} 5$ $\mathrm{mg} / \mathrm{mL}$ 的 MTT, 继续培养 $4 \mathrm{~h}$, 弃去上层液, 加入 100 $\mu \mathrm{L}$ DMSO，振荡 $2 \mathrm{~min}$, 酶标仪 $490 \mathrm{~nm}$ 波长下测定吸光 度. 根据公式计算细胞存活率. 计算公式如下:

细胞存活率 $(\%)=\left(\mathrm{OD}_{\mathrm{T}}-\mathrm{OD}_{\mathrm{B}}\right) /\left(\mathrm{OD}_{0}-\mathrm{OD}_{\mathrm{B}}\right) \times 100 \%$ 其中, $\mathrm{OD}_{\mathrm{T}}$ 为测试化合物的吸光度值, $\mathrm{OD}_{\mathrm{B}}$ 为调零孔吸 光度值, $\mathrm{OD}_{0}$ 为空白对照组吸光度值.

辅助材料(Supporting Information) 目标化合物的核 磁共振谱图和抗菌曲线数据. 这些材料可以免费从本刊 网站(http://sioc-journal.cn/)上下载.

\section{References}

[1] Zhdankin, V. V.; Stang, P. J. Chem. Rev. 2008, 108, 5299.

[2] Chen, H.; Han, J.; Wang L. Angew. Chem., Int. Ed. 2018, 57, 12313.

[3] Zhang, Y.; Han, J.; Liu, Z.-J. RSC Adv. 2015, 5, 25485.

[4] Kuik, W.-J.; Kema, I. P.; Brouwers, A. H.; Zijlma, R.; Neumann, K. D.; Dierckx, R. A. J. O.; DiMagno, S. G.; Elsinga, P. H. J. Nucl. Med. 2015, 56, 106.

[5] DeSolms, S. J.; Woltersdorf, O. W., Jr.; Cragoe, E. J., Jr.; Watson, L. S.; Fanelli, G. M., Jr. J. Med. Chem. 1978, 215, 437.

[6] Das, P.; Etsuko T.; Tokunaga, H.; Akiyama, H.; Doi, H.; Saito, N.; Shibata, N. Beilstein J. Org. Chem. 2018, 14, 364.

[7] Goldstein, E. J. C.; Citron, D. M.; Warren, Y.; Merriam, C. V.; Tyrrell, K.; Fernandez, H.; Radhakrishnan, U.; Stang, P. J.; Conrads, G. Antimicrob. Agents Chemother. 2004, 48, 2766.

[8] Lu, J.; Risbood, P.; Kane, C. T., Jr.; Hossain, M. T.; Anderson, L.; Hill, K.; Monks, A.; Wu, Y.; Antony, S.; Juhasz, A.; Liu, H.; Jiang, G.; Harris, E.; Roy, K.; Meitzler, J. L.; Konaté, M.; Doroshow, J. H. Biochem. Pharmacol. 2017, 143, 25.

[9] Hui, F.; Debiemme-Chouvy, C. Biomacromolecules 2013, 14, 585.

[10] Dong, A.; Wang, Y.-J.; Gao, Y.; Gao, T.; Gao G. Chem. Rev. 2017, 117,4806

[11] Gottardi, W.; Debabov, D.; Nagl, M. Antimicrob. Agents Chemother. 2013, 57, 1107.

[12] Fang, G.; Li, W.; Shen, X.; Perez-Aguilar, J. M.; Chong, Y.; Gao, X.;; Chai, Z.; Chen, C.; Ge, C.; Zhou, R. Nat. Commun. 2018, 9, 129.

[13] Hoque, J.; Akkapeddi, P.; Yarlagadda, V.; Uppu. D. S. S. M.; Kumar, P.; Haldar, J. Langmuir 2012, 28, 12225.

[14] Li, Y.-K.; Liu, B.; Zhang, L.-J.; Zhu, J.-H.; Wan, C.-P.; Mao, Z.-W. Chin. J. Org. Chem. 2018, 38, 949 (in Chinese). (黎勇坤, 刘 蓓, 张丽君, 朱加洪, 万春平, 毛泽伟, 有机化学, 2018, 38, 949.) 